\title{
Sistema de monitoreo de crecimiento de lunares para la detección temprana de cáncer de piel tipo melanoma basado en dispositivos móviles Android
}

Leonel Rosas Arias, Jesús Daniel Rojas Cid, Daniel Benito Román Ocampo, José António López Tello, Waldemar Pérez Bailón

\author{
Instituto Tecnológico de Lázaro Cárdenas, Departamento de Sistemas \\ Computacionales, Cd. Lázaro Cárdenas, Michoacán, México \\ \{rosas.leonel95, danielbenito.roman\}@gmail.com, \\ drcid99@yahoo.com, \{ltello021, waldemar1953\}@hotmail.com
}

\begin{abstract}
Resumen. El cáncer de piel de tipo melanoma es altamente curable si se detecta en una etapa temprana. El melanoma puede surgir en lunares expuestos a radiación UV por periodos prolongados de tiempo, cambiando su color, forma y tamaño. En este trabajo se presenta un sistema para el monitoreo del crecimiento y la apariencia de lunares en la piel como alerta de posible melanoma maligno. Primero, se realiza la segmentación de lunares en la imagen obtenida por el dispositivo movil haciendo uso de filtros en el espacio de color YCbCr. Después, se elimina el ruido restante en la imagen y se calcula el área del lunar. Finalmente, la fotografía, fecha de captura y área son almacenados en una base de datos SQL local para su posterior consulta. Los resultados muestran que al usar una referencia de longitud dada por el usuario se obtiene una exactitud promedio del $95.5 \%$ respecto al área real del lunar.
\end{abstract}

Palabras clave: procesamiento de imágenes, dispositivos móviles, segmentación de color, cáncer de piel de tipo melanoma, software de seguimiento médico.

\section{An Android Mobile Device-based Mole Growth Monitoring System for Early Detection of Melanoma Skin Cancer}

\begin{abstract}
Melanoma skin cancer is highly curable if it is detected in early stages. Melanoma can arise in moles exposed to UV radiation for extended periods of time, changing its color, shape and size. In this paper, we present a monitoring system for mole's growth and appearance as an alert for possible malignant melanoma. First, we segment moles in the image taken by the mobile device by using filters in the $\mathrm{YCbCr}$ color space. Then, we remove residual noise in the image and calculate the mole area. Finally, the photograph, as well as the capture date and the area
\end{abstract}


are stored in a local SQL database for future consultation. Experimental results show that if a reference length is given by the user, the proposed system reaches an average accuracy of $95.5 \%$ respect to the real mole area.

Keywords: image processing, mobile devices, color segmentation, melanoma skin cancer, medical follow-Up software.

\section{Introducción}

El cáncer de piel afecta a personas de todas las razas y grupos étnicos. El grado de detección de cáncer de piel en hombres hispanos se da en menor medida que en los hombres blancos no-hispanos. Por esta razón, los hombres hispanos son más propensos a ser diagnosticados con etapas avanzadas de cáncer [1,2]. El riesgo de desarrollar cáncer de piel se debe a la combinación de factores genéticos y ambientales [3]. La causa más común es la exposición prolongada a la luz ultravioleta [4].

El cáncer de piel se divide en melanoma maligno y cáncer de piel no-melanoma (NMSC por sus siglas en inglés) [3,4,5]. El melanoma representa el $4 \%$ de los casos de cáncer y es responsable directo del $75 \%$ de todas las muertes causadas por cáncer de piel [6].

El melanoma puede aparecer en un lunar pre-existente en la piel que se ha vuelto más obscuro o bien ha cambiado en apariencia, aunque es más común encontrarlo como un lunar nuevo o una peca inusual. Es altamente curable si se detecta en una etapa temprana [7] y se desarrolla en dos fases: Fase de crecimiento radial (sobre la epidermis) y fase de crecimiento vertical (hacia la dermis) [8].

Existe un factor genético conocido como el síndrome familiar de melanoma y lunares atípicos (FAMM por sus siglas en ingles). Pacientes con este síndrome son propensos a sufrir la mutación del gen CDKN2A, causante de melanoma [9]. Los niños que presentan esta condición son mucho más propensos a desarrollar este tipo de cáncer en edades tempranas [5] y por esta razón se recomiendan examinaciones caseras de manera regular, así como tener buenas prácticas de protección solar [10].

Existen diversos parámetros para diagnosticar melanoma. Algunos de estos son: Asimetría, irregularidad en los bordes, variación de color y diámetro, este último es controversial puesto que existen melanomas que son menores a $6 \mathrm{~mm}$ de diámetro [4]. Sin embargo, estudios recientes demuestran que sigue siendo un parámetro útil para diferenciarlos [11].

El cuidado y prevención del melanoma en México está por debajo de lo óptimo. A pesar de que el tratamiento es similar al de países desarrollados, se cree que la razón principal de un diagnóstico en etapas avanzadas es debido a la baja cultura de salud en la población. La mayoría de pacientes con melanoma no acuden a consulta médica hasta que los tumores son grandes, generan incomodidad o ya están causando un problema para su salud [12]. 
Detectar melanoma en pacientes con diversos lunares o lesiones melanocíticas benignas puede ser algo complicado incluso para los más experimentados dermatólogos [13,14]. El mapeo de lunares (del inglés Mole Mapping) es una técnica que involucra tecnología de imagen no invasiva para mejorar el monitoreo de lesiones melanocíticas nuevas o cambiantes. Tecnologías no invasivas de imagen han mostrado detectar melanoma en etapa temprana. El seguimiento médico digital es una tendencia que está revolucionando la manera en la que los pacientes participan en el cuidado y monitoreo de la salud [13].

Las aplicaciones para smartphone (apps) ofrecen la posibilidad de llevar un monitoreo dermatológico periódico. Existen diversas apps para la detección del cáncer de piel pero muy pocas han sido evaluadas como clínicamente eficientes. El $33 \%$ de las apps menciona explícitamente el uso de dermatólogos certificados, el $31 \%$ mencionan la intervención de un equipo médico, mientras que el $36 \%$ no proporciona ningún tipo de información [15]. La mayoría de usuarios que descargan las apps no las usan regularmente, sin embargo, existen esfuerzos para generar conciencia en la población [16]. También se han realizado esfuerzos para enseñar a fisioterapeutas a identificar indicios de cáncer de piel en sus pacientes [17].

Algunas apps desarrolladas para llevar un seguimiento de lunares mediante fotografias son: UMSkin-Check, LoveMySkinMole, FotoSkin y Embarrassing Bodies -- My MoleChecker [15].

Trabajos recientes en sistemas para la detección de melanoma se describen en $[18,19,20]$ obteniéndose resultados alentadores. En [20] se presenta el desarrollo de una app móvil capaz de identificar cáncer de piel en tiempo real utilizando algoritmos automatizados, sin embargo, los resultados de efectividad oscilan entre el $75 \%$ y el diagnóstico toma alrededor de 15 segundos.

Trabajos en el área tele-dermatológica también han utilizado teléfonos móviles con cámara y acceso a internet para facilitar el diagnóstico de un dermatólogo de manera remota [21].

\section{Segmentación de lunares en el espacio de color $\mathrm{YCbCr}$}

La segmentación de objetos mediante su color ha sido uno de los enfoques más comúnmente utilizados para la detección de objetos, trabajos relacionados se muestran en $[22,23,24,25]$. Para realizar esta tarea es posible utilizar diferentes espacios de color, tales como el espacio RGB, HSV, YCbCr, LAB, entre otros.

En este trabajo se ha decidido utilizar el espacio de color $\mathrm{YCbCr}$ debido a que, en base a experimentos realizados, este fue el espacio de color con el que se ha obtenido una segmentación exitosa de manera rápida e intuitiva.

Una imagen representada en el espacio de color YCbCr se expresa como la composición de tres canales:

$$
I_{Y C b C r}=\left(I_{Y}, I_{C b}, I_{C r}\right),
$$


donde $I_{Y}$ es el canal correspondiente a la luma y $I_{C b}$ y $I_{C r}$ son los componentes de crominancia azul y crominancia roja, respectivamente. Esto se ilustra en la Figura 1.

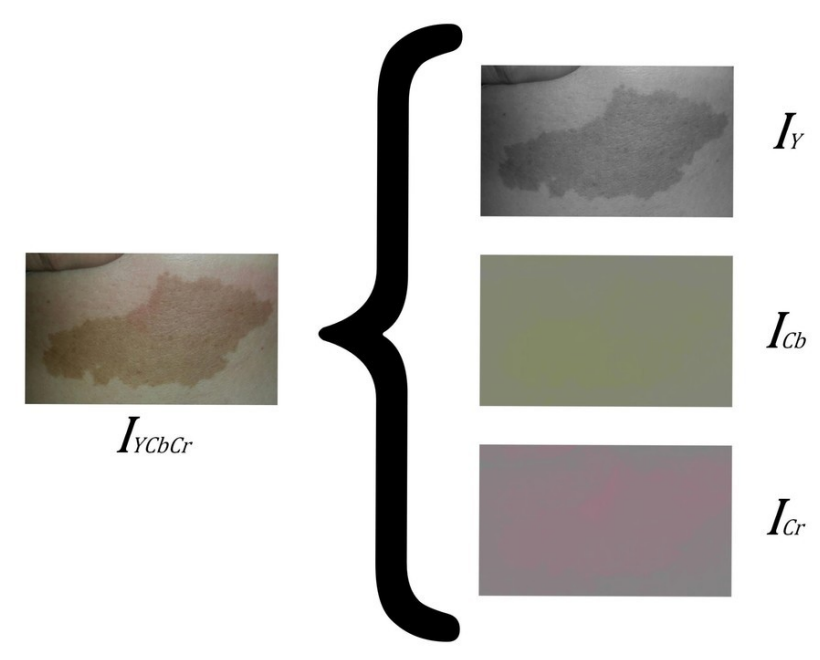

Fig. 1. Componentes de la imagen en el espacio de color $\mathrm{YCbCr}$.

Las imágenes obtenidas por la cámara del dispositivo, haciendo uso de la librería OpenCV, son obtenidas en formato RGBA, donde $\mathrm{R}$ indica el canal rojo; $\mathrm{G}$, el canal verde; $\mathrm{B}$, el canal azul; y $\mathrm{A}$, el canal alfa, correspondiente a la transparencia de la imagen. Para realizar la operación de segmentación en el espacio de color $\mathrm{YCbCr}$, se realiza la conversión de los tres primeros canales de la imagen, es decir de RGB a YCbCr. Esto se obtiene mediante el siguiente modelo:

$$
\begin{gathered}
Y=0,299 R+0,587 G+0,114 B, \\
C b=0,564(B-Y)+\Delta, \\
C r=0,713(R-Y)+\Delta,
\end{gathered}
$$

donde

$$
\Delta= \begin{cases}128 & \text { para imágenes de } 8 \text { bits } \\ 32768 & \text { para imágenes de } 16 \text { bits } \\ 0,5 & \text { para imágenes de punto flotante }\end{cases}
$$


La segmentación de los lunares en el espacio de color $\mathrm{YCbCr}$ se realiza mediante una imagen máscara. Esta imagen contiene la localización de todos los pixeles en la imagen original que pertenecen al lunar. La imagen máscara es una imagen de tipo binaria que está dada por la siguiente condición:

$$
I_{\text {Mask }}(x, y)= \begin{cases}1 & C_{Y} \wedge C_{C b} \wedge C_{C r} \\ 0 & \text { En cualquier otro caso }\end{cases}
$$

donde

$$
\begin{gathered}
C_{Y}: Y_{\min } \leq I_{\text {Mask }_{Y}}(x, y) \leq Y_{\max }, \\
C_{C b}: C b_{\min } \leq I_{\text {Mask }_{C b}}(x, y) \leq C b_{\max }, \\
C_{C r}: C r_{\min } \leq I_{\text {Mask }_{C r}}(x, y) \leq C r_{\max } .
\end{gathered}
$$

$Y_{\min }$ y $Y_{\max }$ son los valores mínimo y máximo del filtro para el canal Y. De la misma manera, los parámetros $C b_{\min }, C b_{\max }, C r_{\min }$ y $C r_{\max }$ representan los valores mínimos y máximos del filtro en sus respectivos canales.

La Figura 2 muestra el resultado de la segmentación de un lunar dorsal perteneciente a uno de los sujetos de prueba. Debido a que la tonalidad de color del lunar es muy similar a algunas otras secciones de la imagen, se producen falsos positivos. En la siguiente sección se aborda la metodología utilizada para eliminar estos falsos positivos con la intervención del usuario.

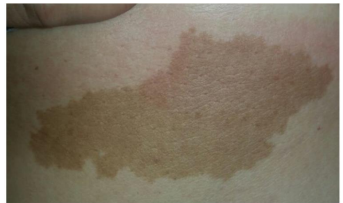

(a)

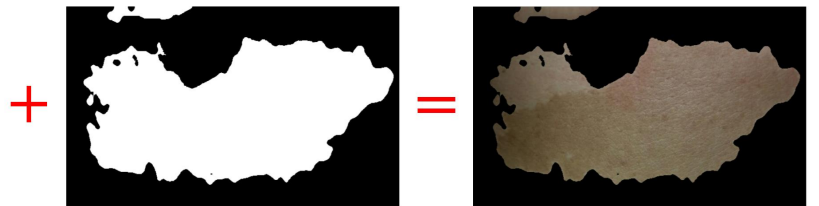

(b) (c)

Fig. 2. Resultado de la segmentación del lunar. a) Imagen original. b) Imagen máscara. c) Imagen segmentada.

\section{Post-procesamiento}

En esta sección se presenta la metodología utilizada para eliminar los pixeles falsos positivos en la segmentación del lunar, es decir, aquellos pixeles que, a pesar de haber pasado el filtro descrito en la sección anterior, no pertenecen al lunar. Este post-procesamiento es opcional, ya que, en algunos casos, el lunar 
puede tener una tonalidad de color muy distinta en comparación a la piel, lo que permite una rápida segmentación sin la necesidad de llevarlo a cabo.

Se presentan dos métodos para resolver este problema: La eliminación de pixeles falsos positivos de manera selectiva y el rellenado dinámico de huecos en los lunares.

\subsection{Eliminación de ruido en la imagen mediante gestos táctiles}

El ruido generado por los pixeles falsos positivos puede ser eliminado selectivamente con ayuda del usuario. Para ello se implementa una estrategia de borrado similar al de los editores de imágenes. Se detecta la posición donde el usuario ha tocado la imagen y se dibuja un circulo de radio:

$$
r_{\text {circle }}=\frac{I_{w i d t h}}{\text { rel }}
$$

donde $I_{\text {width }}$ es el ancho de la imagen en pixeles, y rel es un factor de escala. En este caso se ha hecho uso de $r e l=20$, para una relación de radio veinte veces menor al ancho máximo de la imagen.

El radio del círculo se establece de manera dinámica debido a que la resolución de la imagen mostrada en pantalla varía en relación a la resolución de la pantalla del dispositivo. Un valor de radio fijo podría causar alguna de a las siguientes situaciones:

- En un dispositivo con pantalla de mayor resolución a la del dispositivo de prueba, se borrará una región circular muy pequeña.

- En un dispositivo con pantalla de menor resolución a la del dispositivo de prueba, se borrará una región circular muy grande.

Este círculo coloca todos los pixeles dentro sí mismo a cero. Al colocar todos los pixeles a cero en los tres canales de la imagen, se elimina el ruido en la imagen. Este proceso se ilustra en la Figura 3.

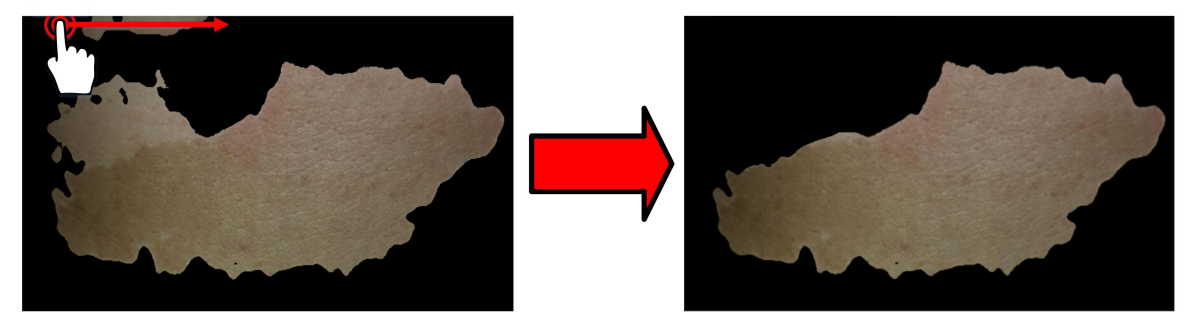

Fig. 3. Segmentación final del lunar con asistencia del usuario. 


\subsection{Rellenado dinámico de huecos en la imagen}

Frecuentemente, al realizar el proceso de segmentación por color existen regiones o huecos dentro del objeto segmentado que no son detectados como parte del mismo. Esto se debe principalmente al reflejo de la iluminación o bien porque existen otros elementos de diferente color dentro del elemento de interés. Estos huecos producidos en el proceso de segmentación pueden ser rellenados para segmentar el objeto de interés en su totalidad. Para ello se utiliza una operación de cerradura morfológica entre la imagen binaria $A$ (correspondiente a la imagen segmentada) y un elemento estructural $B$, es decir:

$$
A \bullet B=(A \oplus B) \ominus B
$$

donde $\oplus$ y $\ominus$ indican un proceso de dilatación y erosión, respectivamente.

En base a resultados experimentales, se propone realizar diez procesos de dilatación seguido de diez procesos de erosión. En cada uno de estos procesos se utiliza un elemento estructural de tipo rectangular de tamaño $K \mathrm{x} K$, en donde $K$ se ha establecido de la siguiente manera:

$$
K= \begin{cases}3 & I_{\text {width }} \leq 300 \\ \text { roundToNextOdd }\left(\frac{I_{\text {width }}}{100}\right) & \text { En cualquier otro caso }\end{cases}
$$

A su vez, la función roundToNextOdd evita que el elemento estructural posea un tamaño par o racional. Un kernel de tamaño par o racional produce errores al usar las funciones de dilatación y erosión de la librería OpenCV. La función roundToNextOdd redondea el valor del argumento y posteriormente aproxima el resultado al siguiente número impar en caso de no serlo. Este proceso se ilustra en la Figura 4.

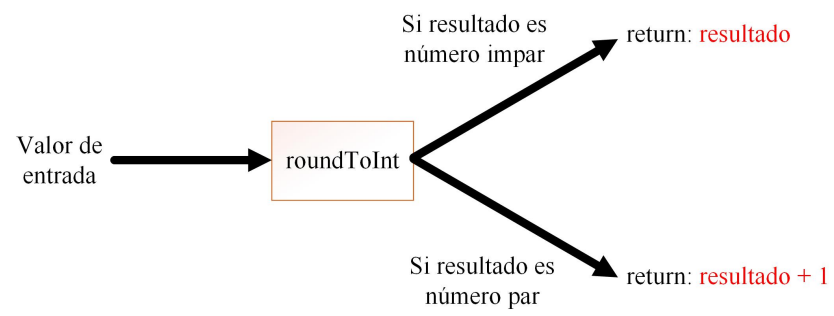

Fig. 4. Estructura interna de la funcion roundToNextOdd.

El proceso de llenado de huecos se ilustra en la Figura 5. Se han añadido diversos huecos de diferentes tamaños en la imagen segmentada del proceso anterior para comprobar la efectividad del método propuesto. 

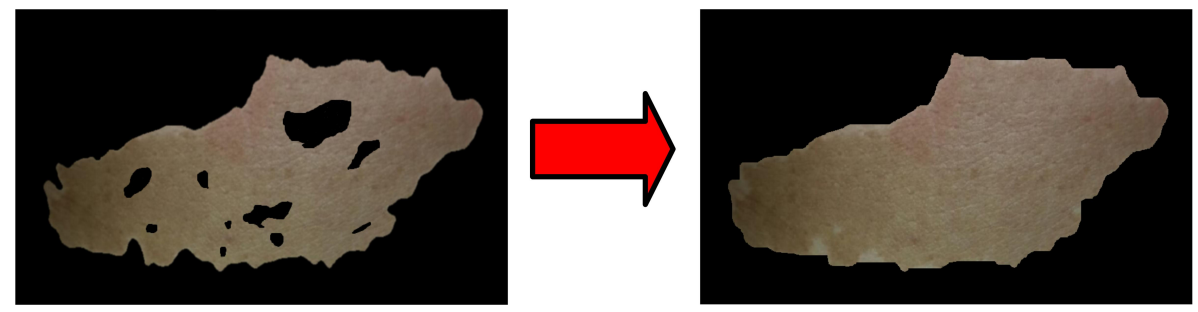

Fig. 5. Rellenado de huecos en la segmentación del lunar.

\section{Determinación del área del lunar}

La determinación del área del lunar se realiza contando todos los pixeles con valor diferente de cero en la imagen segmentada. Este método, aunque bastante fácil de aplicar, presenta un problema significativo. La distancia a la que se toma la fotografía del lunar debe ser consistente para evitar errores en la medición del área. No tomar la fotografía a una distancia consistente en todas las muestras supone alguno de los siguientes problemas:

- Al tomar la fotografía a mayor distancia, menor área es detectada (Un número menor de pixeles representa la misma área del lunar).

- Al tomar la fotografía a menor distancia, mayor área es detectada (Un número mayor de pixeles representa la misma área del lunar).

Para resolver este problema se pide al usuario proporcionar una medida de referencia entre dos puntos de la imagen. Estos dos puntos se alinean con dos líneas de guía verticales visibles durante el proceso de la captura de la imagen. Este proceso se ilustra en la Figura 6 , donde la posición sobre el eje $x$ de las líneas $L_{1}$ y $L_{2}$ son establecidas mediante:

$$
\begin{aligned}
& L_{1}=\frac{3\left(I_{w i d t h}\right)}{8}, \\
& L_{2}=\frac{5\left(I_{w i d t h}\right)}{8} .
\end{aligned}
$$

La posición de las líneas $L_{1}$ y $L_{2}$ puede ser modificada por el usuario durante el proceso de captura de la imagen. Esto se describe en la siguiente sección.

Una vez establecida una medida de referencia y conociendo las posiciones de $L_{1}$ y $L_{2}$ se calcula el área del lunar como:

$$
A=N_{\text {pixels }}\left(\frac{r e f}{L_{2}-L_{1}}\right)^{2},
$$

donde ref es la medida de referencia en milímetros y $N_{\text {pixels }}$ es el número de pixeles diferentes de cero en la imagen. 


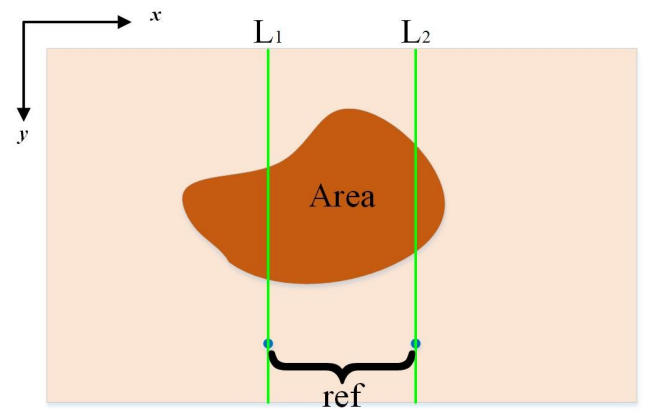

Fig. 6. Determinación del área del lunar con medida de referencia conocida.

\section{Crecimiento del lunar}

Al realizar un chequeo periódico del crecimiento de lunares es deseable establecer una métrica que indique el grado de crecimiento de los mismos. Para este fin, se propone utilizar la pendiente de la recta que mejor se ajusta a los datos históricos del área del lunar. Esta recta es obtenida mediante regresión lineal por el método de mínimos cuadrados. Los parámetros de la recta $y=m x+b$ son determinados mediante:

$$
\begin{gathered}
m=\frac{\sum_{i=0}^{N-1}\left(\mathbf{x}_{i}-\bar{x}\right)\left(\mathbf{y}_{i}-\bar{y}\right)}{\sum_{i=0}^{N-1}\left(\mathbf{x}_{i}-\bar{x}\right)^{2}}, \\
b=\bar{y}-m(\bar{x}),
\end{gathered}
$$

donde $\mathbf{x}$ es el vector correspondiente al área del lunar, $\mathbf{y}$ el vector correspondiente al número de muestra, $\bar{x}$ y $\bar{y}$ son la media cada uno de los vectores y $N$ es el número de muestras.

El valor de la pendiente de la recta proporciona una referencia del crecimiento del lunar. Adicionalmente, el signo de la pendiente también proporciona información. Un signo positivo, indicaría un incremento del área lunar, mientras que, un signo negativo indicaría la reducción del mismo.

\section{Resultados}

En esta sección se presenta la aplicación desarrollada y se da una descripción de los elementos que la componen. También se presenta el desempeño del sistema en la medición de área de los lunares ante diversas condiciones. Las pruebas de desempeño del sistema se realizaron en un teléfono celular móvil Samsung Galaxy $S 5$. Por otra parte, las pruebas de compatibilidad de software, se realizaron adicionalmente en los siguientes dispositivos: Samsung Galaxy S7, ZTE Blade 
Leonel Rosas Arias, Jesús Daniel Rojas Cid, Daniel Benito Román Ocampo, et al.

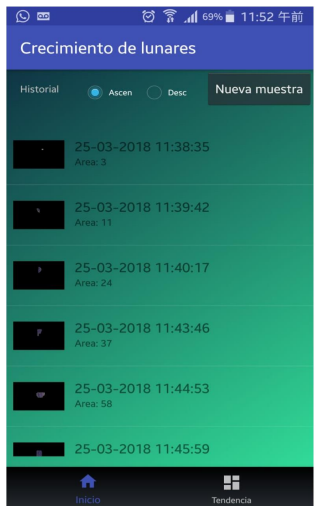

(a)

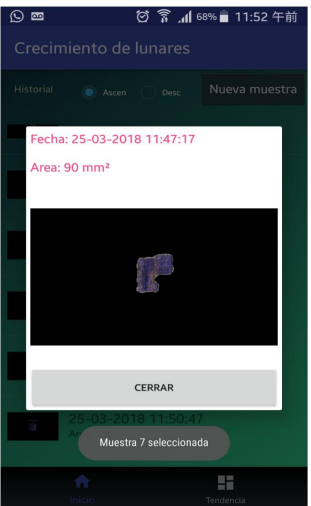

(b)

Fig. 7. Módulo principal. a) Visualización general de las muestras. b) Visualización individual.

v6, Alcatel Pixi 4, Samsung Galaxy Grand Prime y Samsung Galaxy Grand Prime Plus.

\subsection{Aplicación móvil}

La aplicación está dividida en tres módulos:

- Módulo principal. Este módulo, mostrado en la Figura 7, despliega un listado de las muestras obtenidas que pueden ser ordenadas por fecha, de la más reciente a la más antigua y viceversa. Se muestra la fecha de captura, el área del lunar y la vista previa de la fotografía del lunar para cada una de las muestras. Al presionar sobre alguno de los elementos se muestra la fotografía en gran tamaño junto con sus datos correspondientes.

- Módulo de obtención de nueva muestra. En este módulo se realiza la adquisición, procesamiento y post-procesamiento de la imagen del lunar. Proporciona los controles necesarios para modificar los parámetros del filtro en el espacio de color YCbCr y la posición de las líneas de guía. Además, una vez adquirida la imagen, proporciona opciones para rellenar los posibles huecos de la segmentación y para deshacer el último cambio realizado. También se proporciona al usuario la posibilidad de eliminar el ruido de la imagen segmentada mediante gestos táctiles. Este módulo se muestra en la Figura 8.

- Módulo del historial gráfico. Este módulo muestra el gráfico del comportamiento del crecimiento del lunar. El gráfico muestra el número de muestra en el eje $x$ y el área del lunar en el eje $y$. También se muestra la recta $y=m x+b$ obtenida mediante regresión lineal que indica el crecimiento del lunar. Al tocar sobre alguna de las muestras del gráfico, se presenta un mensaje con su información respectiva. Esto se aprecia en la Figura 9. 
Sistema de monitoreo de crecimiento de lunares para la detección temprana de cáncer de piel...

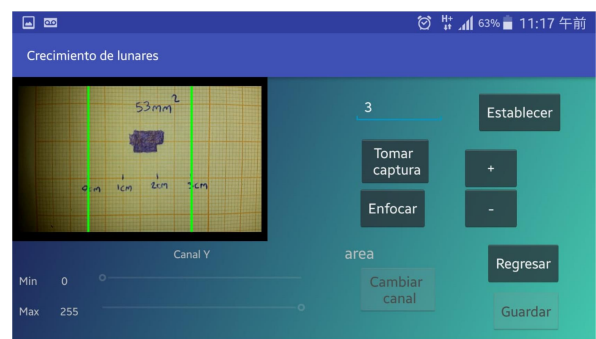

(a)

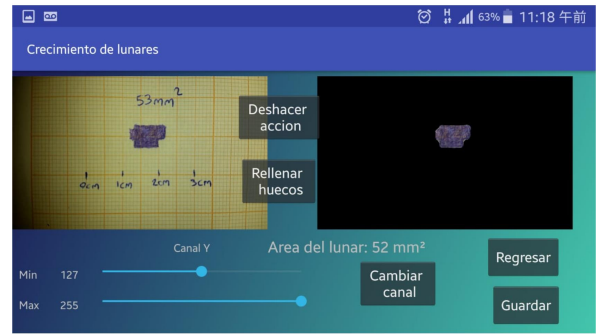

(b)

Fig. 8. Módulo de obtención de nueva muestra. a) Adquisición de la imagen. b) Segmentación y guardado.

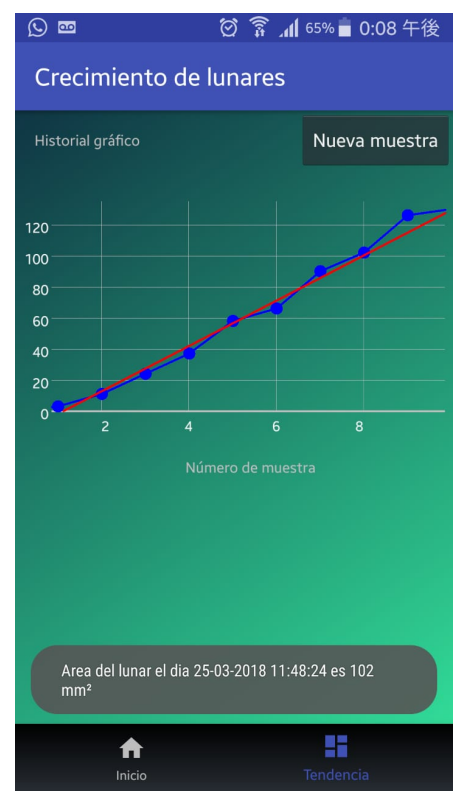

Fig. 9. Módulo del historial gráfico. 


\subsection{Desempeño del sistema en la medición de área}

Para probar la efectividad de la medición de área del sistema se han utilizado hojas milimétricas. Estas hojas cuadriculadas con cuadros de $1 \mathrm{~mm}^{2}$ son ideales para conocer de antemano el área de las superficies de prueba al contar los cuadros individuales. En las hojas se han dibujado superficies con un área aleatoria de entre 0 y $150 \mathrm{~mm}^{2}$. Se han realizado 30 pruebas en 10 diferentes medidas de superficie. Las pruebas se realizaron con tres medidas diferentes de referencia previamente establecidas. Se ha determinado mediante el promedio del error de todas las muestras que la exactitud del sistema es de aproximadamente el 95,5\%. El resultado de estas pruebas se muestra en el gráfico de la Figura 10.

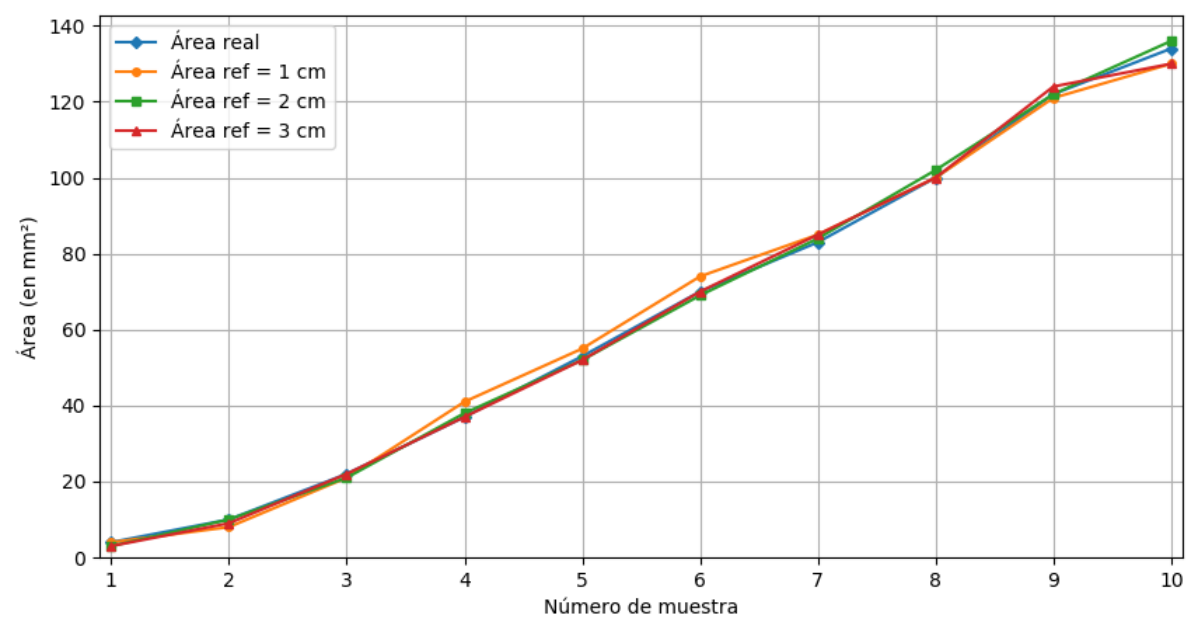

Fig. 10. Desempeño del sistema con diferentes medidas de referencia.

\section{Conclusiones y trabajos futuros}

En este trabajo se ha presentado una herramienta para el monitoreo y detección temprana de cáncer de piel del tipo melanoma a partir del crecimiento de lunares. Se ha presentado un método de segmentación rápida de lunares mediante su color haciendo uso de filtros en el espacio de color $\mathrm{YCbCr}$.

Existen casos en donde el color de la piel es muy similar al color del lunar, por lo que, es necesario un mayor nivel de interacción con el usuario para eliminar las regiones de la imagen que no pertenecen al lunar. Este proceso de corrección manual, aunque reduce el grado de automatización del sistema, puede realizarse sin mayor dificultad de manera rápida. La determinación del área ha sido realizada exitosamente habiendo obtenido resultados de hasta el $100 \%$ de exactitud respecto al área real del lunar. El margen de error por medición ha sido 
de un par de pixeles, por lo que, en lunares con superficies pequeñas (menores a $10 \mathrm{~mm}^{2}$ ) los errores porcentuales tienden a incrementar en comparación con lunares de superficies mayores. La determinación del área del lunar se ha visto afectada por diversos factores. Los dos factores principales son: La distorsión en la imagen causada por el lente de la cámara del dispositivo y el ángulo de captura de la imagen. Este último se presenta cuando la fotografía no es tomada de manera paralela a la superficie de la piel. A pesar de estos factores de error, el porcentaje de efectividad promedio del sistema ha sido del 95,5\%. Por esta razón, creemos que el sistema ofrece una medida confiable tratándose de un equipo no especializado.

En trabajos futuros se proyecta la adición de nuevas funcionalidades al sistema con el fin de realizar un diagnóstico oportuno de cáncer de piel utilizando técnicas de Machine Learning. Estas nuevas funcionalidades serán capaces de analizar el color, la forma y la textura del lunar de manera local y/o global para diferenciar entre melanoma maligno y otras lesiones melanocíticas benignas.

Agradecimientos. Trabajo de investigación apoyado y financiado por el Tecnológico Nacional de México/Instituto Tecnológico de Lázaro Cárdenas.

\section{Referencias}

1. Zhou, J., Enewold, L., Peoples, G. E., McLeod, D. G., Potter, J. F., Steele, S. R., Clive, K. S., Stojadinovic, A., Zhu, K.: Colorectal Prostate Skin Cancer Screening Among Hispanic White Non-Hispanic Men 2000-2005. Journal of the National Medical Association, 103(4), pp. 343-350 (2011)

2. Agbai, O. N., Buster, K., Sanchez, M., Hernandez, C., Kundu, R. V., Chiu, M., Roberts, W. E., Draelos, Z. D., Bhushan, R., Taylor, S. C., Lim, H. W.: Skin cancer and photoprotection in people of color: A review and recommendations for physicians and the public. Journal of the American Academy of Dermatology, 70(4), pp. 748-762 (2014)

3. Chummun, S., McLean, N. R.: The management of malignant skin cancers. Surgery (Oxford), 35(9), pp. 519-524 (2017)

4. Craythorne E., Al-Niami F.: Skin cancer. Medicine, 45(7), pp. 431-434 (2017)

5. Maguire-Eisen, M.: Skin Cancer: A Growing Health Problem for Children. Seminars in Oncology Nursing, 29(3), pp. 206-213 (2013)

6. Godoy, S. E., Ramirez, D. A., Myers, S. A., Winckel, G., Krishna, S., Berwick, M., Padilla, R. S., Sen, P., Krishna S.: Dynamic infrared imaging for skin cancer screening. Infrared Physics \& Technology, 70, pp. 147-152 (2015)

7. Reshma, M., Priestly Shan, B.: Review of Image Acquisition and Classification Methods on Early Detection of Skin Cancer. Proceedings of Fifth International Conference on Soft Computing for Problem Solving, pp. 1015-1025 (2016)

8. Holte, K., Biswas, A.: Pathology of malignant skin tumours. Surgery (Oxford), 35(9), pp. 478-483 (2017)

9. Soura, E., Eliades, P. J., Shannon, K., Stratigos, A. J., Tsao H.: Hereditary melanoma: Update on syndromes and management: Genetics of familial atypical multiple mole melanoma syndrome. Journal of the American Academy of Dermatology, 74(3), pp. 395-407 (2016) 
10. Hawryluk, E. B., Liang, M. G.: Pediatric Melanoma, Moles, and Sun Safety. Pediatric Clinics of North America, 61(2), pp. 279-291 (2014)

11. Glazer, A. M., Rigel, D. S., Winkelmann, R. R., Farberg, A. S.: Clinical Diagnosis of Skin Cancer: Enhancing Inspection and Early Recognition. Dermatologic Clinics, 35(4), pp. 409-416 (2017)

12. Lino-Silva, L. S., Domínguez-Rodríguez, J. A., Aguilar-Romero, J. M., MartínezSaid, H., Salcedo-Hernández, R. A., García-Pérez, L., Herrera-Gómez, A., CuellarHubbe, M.: Melanoma in Mexico: Clinicopathologic Features in a Population with Predominance of Acral Lentiginous Subtype. Annals of Surgical Oncology, 23(13), pp. 4189-4194 (2016)

13. Berk-Krauss, J., Polsky, D., Stein, J. A.: Mole Mapping for Management of Pigmented Skin Lesions. Dermatologic Clinics, 35(4), pp. 439-445 (2017)

14. Bresler, S. C., Wanat, K., Seykora, J.: Benign Melanocytic Lesions and Melanoma. Pathobiology of Human Disease, pp. 1182-1192 (2014)

15. Chao, E., Meenan, C. K., Ferris, L. K.: Smartphone-Based Applications for Skin Monitoring and Melanoma Detection. Dermatologic Clinics, 35(4), pp. 551-557 (2017)

16. Noar, S. M., Leas, E., Althouse, B. M., Dredze, M., Kelley, Ayers, J. W.: Can a selfie promote public engagement with skin cancer?. Preventive Medicine (2017)

17. Trotter, S. C., Louie-Gao, Q., Hession, M. T., Cummins, D.: Skin Cancer Education for Massage Therapists: A Novel Approach to the Early Detection of Suspicious Lesions. Journal of Cancer Education, 29(2), pp. 266-269 (2014)

18. Kostopoulos, S. A., Asvestas, P. A., Kalatzis, I. K., Sakellaropoulos, G. C., Sakkis, T. H., Cavouras, D. A., Glotsos, D. T.: Adaptable pattern recognition system for discriminating Melanocytic Nevi from Malignant Melanomas using plain photography images from different image databases. International Journal of Medical Informatics, 105, pp. 1-10 (2017)

19. Dalila, F., Zohra, A., Reda, K., Hocine, C.: Segmentation and classification of melanoma and benign skin lesions. Optik - International Journal for Light and Electron Optics, 140, pp. 749-761 (2017)

20. Taufiq, M. A., Hameed, N., Anjum, A., Hameed, F.: m-Skin Doctor: A Mobile Enabled System for Early Melanoma Skin Cancer Detection Using Support Vector Machine. eHealth $360^{\circ}$, pp. 468-475 (2017)

21. Walocko, F. M., Tejasvi, T.: Teledermatology Applications in Skin Cancer Diagnosis. Dermatologic Clinics, 35(4), pp. 559-563 (2017)

22. Garcia-Lamont, F., Cervantes, J., López, A., Rodriguez, L.: Segmentation of images by color features: A survey. Neurocomputing, 292, pp. 1-27 (2018)

23. Hassanat, A. B. A., Alkasassbeh, M., Al-awadi, M., Alhasanat, E. A. A.: Colorbased object segmentation method using artificial neural network. Simulation Modelling Practice and Theory, 64, pp. 3-17 (2016)

24. Sikha, O. K., Kumar, S. S., Soman, K. P.: Salient region detection and object segmentation in color images using dynamic mode decomposition. Journal of Computational Science (In Press) (2017)

25. Cernadas, E., Fernández-Delgado, M., González-Rufino, E., Carrión, P.: Influence of normalization and color space to color texture classification. Pattern Recognition, 61, pp. 120-138 (2017) 